\title{
Eficacia del tratamiento conservador mediante rehabilitación del piso pélvico en mujeres con incontinencia urinaria de esfuerzo
}

Aranda-Lozano J, Sierra-Labarta R, Sánchez-Zalabardo D

Resumen

ANTECEDENTES: la incontinencia urinaria de esfuerzo es un padecimiento de gran prevalencia en la mujer. Puesto que el tratamiento farmacológico tiene un papel limitado, la rehabilitación del piso pélvico y la cirugía son los principales recursos terapéuticos.

OBJETIVO: conocer la tasa de curación de mujeres con incontinencia urinaria de esfuerzo incluidas en nuestro protocolo de rehabilitación del piso pélvico.

MATERIALES Y MÉTODOS: estudio analítico, descriptivo, retrospectivo y longitudinal de la rehabilitación del piso pélvico, a lo largo de un año, en pacientes con incontinencia urinaria de esfuerzo.

RESULTADOS: de 56 pacientes que iniciaron la rehabilitación, 48 completaron el programa (tasa de abandono de 14.2\%). A seis meses de la finalización del programa, 18 pacientes $(37.5 \%)$ refirieron estar curadas, 22 (45.8\%) haber mejorado y 8 (16.7\%) no mejoraron.

CONCLUSIÓN: la rehabilitación del piso pélvico mejora clínicamente la incontinencia urinaria de esfuerzo, aumenta la calidad miccional y, en un alto porcentaje, evita la corrección quirúrgica del padecimiento.

PALABRAS CLAVE: incontinencia, esfuerzo, rehabilitación, piso pélvico.

Rev Mex Urol. 2017 January;77(1):12-19.

\section{Efficacy of pelvic floor rehabilitation treatment in women with stress urinary incontinence}

\section{Aranda-Lozano J, Sierra-Labarta R, Sánchez-Zalabardo D}

\section{Abstract}

BACKGROUND: Stress urinary incontinence is a highly prevalent pathology in women. Drug treatment has a limited role, with pelvic floor rehabilitation and surgery being the main therapeutic weapons.
Servicio de Urología, Hospital Reina Sofía de Tudela (Navarra), España.

Recibido: junio 2016

Aceptado: enero 2017

Correspondencia Jorge Aranda Lozano Hospital Reina Sofía, Carretera de Tarazona Km. 3 31500 Tudela, España arandajor@gmail.com

Este artículo debe citarse como

Aranda-Lozano J, Sierra-Labarta R, SánchezZalabardo D. Eficacia del tratamiento conservador mediante rehabilitación del piso pélvico en mujeres con incontinencia urinaria de esfuerzo. Rev Mex Urol. 2017 ene; 77(1):12-19. 
OBJECTIVE: To know the cure rate in women with stress urinary incontinence that underwent our pelvic floor rehabilitation protocol.

MATERIALS AND METHODS: The results of patients with stress urinary incontinence that received conservative treatment through pelvic floor rehabilitation for one year were analyzed through a retrospective, analytic, descriptive study.

RESULTS: Of the 56 patients that began the rehabilitation treatment, 48 completed the program (dropout rate of $14.2 \%$ ). Six months after the program, 18 patients (37.5\%) stated they were cured, 22 patients $(45.8 \%)$ reported improvement, and 8 patients $(16.7 \%)$ said they had no improvement.

CONCLUSIONS: Pelvic floor rehabilitation produces clinical improvement in stress urinary incontinence. There was improvement in micturition quality in our study patients and surgical correction of stress urinary incontinence was not necessary in a high percentage of the cases.

KEY WORDS: Incontinence; Stress; Rehabilitation; Pelvic floor
Servicio de Urología, Hospital Reina Sofía de Tudela (Navarra), España.

Correspondence
Jorge Aranda Lozano
Hospital Reina Sofía, Carretera de Tara-
zona Km. 3
31500 Tudela, España
arandajor@gmail.com

\section{ANTECEDENTES}

La incontinencia urinaria es un padecimiento muy frecuente en nuestro medio. Pueden diferenciarse tres tipos: de esfuerzo, urgencia y mixta; $;^{1,2}$ es mucho más frecuente en la mujer que en el varón. ${ }^{3}$

En general, la prevalencia media estimada de incontinencia urinaria, en cualquiera de sus tipos es de entre 20 y $30 \%$, con incremento incluso de 30 a $40 \%$ en personas de mediana edad y hasta 30 a $50 \%$ en ancianos. En un estudio epidemiológico efectuado en Noruega con 27,938 mujeres mayores de 20 años (estudio EPICONT del año 2000), 25\% de ellas reportaron algún tipo de incontinencia urinaria. En el estudio EPICONT la incontinencia urinaria de esfuerzo representó $49.4 \%$ versus 35 y $10.9 \%$ de la incontinencia mixta y de urgencia, respectivamente. Por grupos de edad, la incontinencia urinaria de esfuerzo es más frecuente (55.1\%) en mujeres de 20-39 años, de $45.4 \%$ en el grupo de $40-54$ años y de $32.4 \%$ en las mayores de 55 años. 4,5

Los principales factores de riesgo de incontinencia urinaria de esfuerzo son: edad, obesidad y paridad. ${ }^{4-10}$ La alteración del equilibrio e integridad de los músculos del piso pélvico es la explicación fisiopatológica más aceptada. ${ }^{11-14}$

El tratamiento farmacológico tiene una participación muy limitada; la duloxetina es el medicamento más estudiado para la incontinencia urinaria de esfuerzo. ${ }^{15,16}$ Respecto al tratamiento conservador, inicialmente deben indicarse medidas higiénico-dietéticas: reducción de peso, ${ }^{17}$ abandono del hábito tabáquico, menor consumo de cafeína y prevención del estreñimiento. ${ }^{18,19}$

Lo principal en el tratamiento conservador es la rehabilitación de la musculatura del piso pélvico, ${ }^{20-25}$ que se refuerza con biofeedback ${ }^{26-29}$ y electroestimulación. ${ }^{30-32}$ Cuando esto fracasa, la 
alternativa es la cirugía correctiva: implantación de cabestrillos suburetrales libres de tensión, tipo TOT (Transobturator Tape) o TVT (Tension-Free Vaginal Tape), entre otras, que no están exentas de complicaciones. ${ }^{33-37}$

El objetivo de este estudio es conocer la tasa de curación en mujeres con incontinencia urinaria de esfuerzo incluidas en nuestro protocolo de rehabilitación del piso pélvico.

\section{MATERIALES Y MÉTODOS}

Estudio analítico, descriptivo, retrospectivo y longitudinal efectuado entre el 1 de marzo de 2014 y el 1 marzo de 2015 en pacientes que durante un año recibieron tratamiento conservador mediante rehabilitación del piso pélvico por incontinencia urinaria de esfuerzo. Criterios de inclusión: mujeres mayores de edad con incontinencia urinaria de esfuerzo pura, incontinencia urinaria mixta o con prolapso de órganos pélvicos asociado con incontinencia urinaria de esfuerzo. Criterio de exclusión: pacientes con otras afecciones del aparato urinario inferior: incontinencia urinaria de urgencia, tumores, litiasis vesicales o cistitis intersticial.

Variables de estudio previas a la rehabilitación: edad, peso, índice de masa corporal (IMC), grado clínico de incontinencia urinaria según la clasificación de Ingelman-Sundberg, tipo de incontinencia urinaria, meses de evolución, prolapso de órganos pélvicos, cantidad de compresas antes de la rehabilitación, antecedentes de hipertensión arterial, diabetes, hábito tabáquico, estreñimiento, menopausia, depresión; antecedentes obstétricos (partos vaginales, cesáreas, abortos), antecedentes quirúrgicos de prolapso y cirugías correctoras de la incontinencia urinaria.

El tratamiento de rehabilitación del piso pélvico consta de 12 sesiones si la incontinencia urinaria de esfuerzo es leve y de 15 sesiones si la incontinencia es moderada o severa. La duración de cada sesión es de 30-40 minutos. El programa de rehabilitación se organiza de la siguiente manera:

1. En la primera sesión se explica en qué consiste el tratamiento de rehabilitación. Se entrega una hoja con recomendaciones higiénico-dietéticas para la incontinencia urinaria: pérdida de peso, evitar: el consumo de café, alcohol, tabaco y esfuerzos intensos; controlar la ingesta de líquidos (hasta 2 litros al día), usar ropa cómoda. Se enseñan los principales ejercicios de Kegel.

2. De la sesión 2 a 6 (semana 1 a 3)

a. Se insiste en la correcta ejecución de los ejercicios de Kegel con la supervisión de un fisioterapeuta, con aumento progresivo del número de repeticiones, de acuerdo con la tolerancia de la paciente.

b. Se realizaba un biofeedback y electroestimulación durante 15-20 minutos con el electroestimulador Myomed 632X (EnrafNonius). El tipo de corriente eléctrica utilizada durante la sesión de biofeedback y electroestimulación es TENS bifásica pulsada y simétrica. Como refuerzo de la correcta ejecución de la contracción se lleva a cabo un miobiofeedback con electrodos vaginales, con registro de la señal electromiográfica de las contracciones, con habilitación de una señal acústica (pitido) cuando el paciente alcanza los valores mínimos establecidos. Gracias al biofeedback vaginal, la paciente consigue identificar y contraer selectivamente los músculos pélvicos.

Para la realización de la electroestimulación se utilizan electrodos vaginales de $2 \mathrm{~mm}$. En incontinencia urinaria de esfuerzo leve se aplica una frecuencia de $70 \mathrm{~Hz}$ con ancho de pulso de 20160 microsegundos. Esos estímulos se dirigen, 
sobre todo, a las fibras fásicas; el tiempo de pausa entre contracciones es el doble del tiempo de estímulo. Si la incontinencia urinaria de esfuerzo es moderada o grave se utiliza una frecuencia de $20 \mathrm{~Hz}$ y ancho de pulso de 320-740 microsegundos, con esos estímulos se trabajan las fibras tónicas. El tiempo de pausa entre contracciones es el doble que el del estímulo.

c. Cuando la paciente lleva a cabo la correcta ejecución de los ejercicios y tiene adecuada conciencia de los mismos, se inician ejercicios de gimnasia hipopresiva.

3. De las sesiones 7 a 15 (semanas 4 a 6 ):

a. Se continúa con el biofeedback y la electroestimulación, se aumenta la duración de las sesiones de electroestimulación hasta los 30 minutos si existe buena tolerancia por parte de la paciente.

b. La dificultad de los ejercicios se incrementa progresivamente.

c. En las últimas sesiones, a las interesadas se les explica el uso diario de las bolas chinas o conos vaginales, con el propósito de mantener el tono muscular adquirido durante las sesiones.

Con el propósito de valorar el resultado, las pacientes se reevalúan en consultas a los seis meses de la finalización del tratamiento de rehabilitación. Se registran las siguientes variables: cantidad de compresas postrehabilitación, resultado de rehabilitación y colocación de TOT. Se considera curación si después del tratamiento de rehabilitación se corrigen por completo los episodios de incontinencia urinaria de esfuerzo y se deja de utilizar compresas (o solo se recurre a una "por seguridad"). Si la paciente consigue disminuir el número de episodios de incontinencia urinaria o del uso de compresas, se considera mejoría. Si la paciente permanece igual que antes del inicio del programa de rehabilitación, es decir, no consiguió reducir el número de episodios de incontinencia urinaria ni de compresas, se considera sin cambios. Por último, si la paciente incrementa su incontinencia urinaria de esfuerzo, se considera empeoramiento.

\section{RESULTADOS}

Se estudiaron 56 pacientes que iniciaron el tratamiento de rehabilitación y lo finalizaron 48 . La tasa de abandono fue de $14.2 \%$; es decir, que 8 pacientes dejaron el programa por falta de motivación o ausencia de mejoría subjetiva.

La edad media de las pacientes fue: 47.5 años (31-74 años), con peso medio de $68.2 \mathrm{~kg}$ (46$100 \mathrm{~kg}$ ) y media de IMC de 25.8 (19.1-38.1). La media de tiempo de evolución de los síntomas de incontinencia urinaria de esfuerzo fue de 41.5 meses (2-360 meses). La media de compresas o material absorbente utilizado por las mujeres antes de iniciar la rehabilitación fue de 2.06 (0-8) compresas al día; 5 mujeres no utilizaban compresas al inicio de la rehabilitación, 9 pacientes usaban una compresa al día y 42 necesitaron 2 o más compresas.

De las 56 pacientes que comenzaron el programa de rehabilitación, $36(64.3 \%)$ tenían incontinencia urinaria de esfuerzo pura, 15 $(26.8 \%)$ incontinencia urinaria mixta y $5(8.9 \%)$ prolapso de órganos pélvicos asociado con incontinencia urinaria de esfuerzo (Cuadro 1).

El grado clínico de incontinencia urinaria de esfuerzo establecido en la primera consulta, según la clasificación de Ingelman-Sundberg, fue: 14 pacientes $(25 \%)$ incontinencia urinaria de esfuerzo leve, $37(66.1 \%)$ incontinencia moderada y 5 $(8.9 \%)$ incontinencia severa (Cuadro 2).

Del total, 7 pacientes (12.5\%) padecían hipertensión y recibían tratamiento farmacológico, 2 (3.5\%) eran diabéticas ( 1 de ellas tipo 1), 16.07\% 
Cuadro 1. Tipos de incontinencia tratados

\begin{tabular}{|l|c|c|}
\hline$(\mathbf{n = 5 6 )}$ & $\mathbf{n}$ & $\%$ \\
\hline Incontinencia de esfuerzo & 36 & 64.3 \\
\hline Incontinencia mixta & 15 & 26.8 \\
\hline Prolapso asociado & 5 & 8.9 \\
\hline
\end{tabular}

Cuadro 2. Grado clínico de la incontinencia

\begin{tabular}{|l|c|c|}
\hline$(\mathbf{n = 5 6 )}$ & $\mathbf{n}$ & $\%$ \\
\hline Incontinencia leve & 14 & 25 \\
\hline Incontinencia moderada & 37 & 66.1 \\
\hline Incontinencia severa & 5 & 8.9 \\
\hline
\end{tabular}

(9 pacientes) eran fumadoras, 12 (21.4\%) padecían o habían padecido algún episodio depresivo y $12(21.4 \%)$ sufrían estreñimiento.

En cuanto a la paridad, 11 mujeres (19.6\%) tuvieron un único hijo por vía vaginal, 22 (39.3\%) tuvieron 2 y 8 (14.2\%) 3 o más hijos. Cinco pacientes $(8.9 \%)$ parieron por cesárea una sola vez y 2 tuvieron dos cesáreas (3.5\%). Ocho (14.2\%) pacientes eran nulíparas.

Por lo que se refiere a los abortos: 12 pacientes (21.4\%) tuvieron uno y 4 (7.1\%) dos.

De todas las pacientes, antes de la rehabilitación 19 (33.9\%) estaban en la postmenopausia. A 6 pacientes $(10.7 \%)$ se les había efectuado histerectomía por miomas y metrorragias antes del inicio de la rehabilitación (ninguna por patología tumoral).

En cuanto a las cirugías de prolapso, 4 pacientes (7.1\%) habían sido intervenidas para reparación del prolapso de órganos pélvicos mediante colposuspensión. Respecto a las cirugías de incontinencia urinaria, 2 pacientes $(3.5 \%)$ habían sido intervenidas por incontinencia mediante cabestrillos TOT, antes del inicio de la rehabilitación.
A los seis meses después de la finalización del programa de rehabilitación, de las 56 pacientes, solo 48 completaron el tratamiento, 18 (37.5\%) refirieron no tener escapes y dejaron de usar compresas; 22 (45.8\%) refirieron disminución de los síntomas (menos episodios de incontinencia urinaria de esfuerzo y de compresas al día); 8 $(16.7 \%)$ declararon no haber mejorado. El $83.3 \%$ de las pacientes se curaron o les disminuyeron los síntomas de incontinencia urinaria de esfuerzo iniciales (Cuadro 3).

Las ocho pacientes que no mejoraron con el tratamiento se llevaron a cirugía de TOT y 5 de las 22 pacientes que habían referido mejoría requirieron, también, colocación de TOT por mejoría insuficiente de la incontinencia. Así, de las 48 pacientes que finalizaron el programa de rehabilitación 27.1\% $(n=13)$ requirieron TOT (Cuadro 3).

\section{DISCUSIÓN}

El tratamiento conservador mediante rehabilitación del piso pélvico constituye una gran arma terapéutica en la incontinencia urinaria de esfuerzo con la que puede conseguirse la curación o, al menos, disminución de los síntomas. En nuestra serie, de las 48 pacientes que completaron el programa $37.5 \% \quad(n=18)$ se curaron (remisión total de la incontinencia urinaria de esfuerzo) y $45.8 \%(n=22)$, refirieron que los síntomas disminuyeron y requirieron menos compresas al día a los seis meses de haber finalizado el programa.

Cuadro 3. Resultados después del programa de rehabilitación

\begin{tabular}{|l|c|c|}
\hline $\mathbf{( n = 4 8 )}$ & $\mathbf{n}$ & $\%$ \\
\hline Curación & 18 & 375 \\
\hline Mejoría & 22 & 45.8 \\
\hline Sin mejoría & 8 & 16.7 \\
\hline No requirieron cirugía transobturadora & 35 & 72.9 \\
\hline Requirieron cirugía transobturadora & 13 & 27.1 \\
\hline
\end{tabular}


En el año 2002, el Hospital Virgen del Camino de Sevilla, España, publicó un estudio con 412 pacientes con incontinencia urinaria de esfuerzo rehabilitadas en un lapso de cuatro años, con los siguientes resultados: éxito $45.9 \%(\mathrm{n}=189)$, resultados aceptables $38.8 \%(n=160)$ y $15.3 \%$ $(n=65)$ fracasos. ${ }^{38}$ No existen muchos estudios con grandes series de pacientes para poder comparar, como referencia, las tasas de éxito, mejoría o fracaso. Nuestra tasa de curación más mejoría (81.5\%) es similar a la del año 2002 del Hospital Virgen del Camino de Sevilla (84.7\%).

El tratamiento conservador evita, en alto porcentaje, la necesidad de intervención quirúrgica. En nuestra serie, 35 pacientes $(72.9 \%)$ no requirieron cirugía para corrección de la incontinencia urinaria de esfuerzo. En 2003 se publicó una revisión sistemática del costo-efectividad de las cirugías de incontinencia urinaria de esfuerzo que se estimó en 1,114 libras esterlinas para TVT y de 1,317 libras esterlinas para otras técnicas de colposuspensión con cabestrillos convencionales. ${ }^{39}$ Un estudio efectuado en el Hospital de Fuenlabrada, en Madrid, España, el gasto por TOT en régimen de CMA sin complicación posquirúrgica fue de 4,923 versus 6,871 euros si se realizaba en régimen de hospitalización. ${ }^{40}$ La rehabilitación no requiere ingreso hospitalario, ni anestesia, ni tratamiento farmacológico posterior; esto implica un ahorro considerable en comparación con la cirugía. El gasto en la rehabilitación se reduce a parches adhesivos y electrodos vaginales para electroestimulación y biofeedback, que es bastante más asequible. ${ }^{41}$

La cirugía no está exenta de complicaciones, lo que añade morbilidad al procedimiento quirúrgico. Las complicaciones más frecuentes, según el momento de su aparición, son: intraoperatoriamente la perforación vesical, complicaciones tempranas de infección y retención urinaria y complicaciones tardías, como el dolor uretral o vaginal, dispareunia y extrusión de la ma-
Ila. ${ }^{42-45}$ En nuestra serie sola una paciente refirió molestias miccionales temporales debidas a la intolerancia a la electroestimulación.

Otro de los factores decisivos a la hora de elegir el tratamiento conservador, es el grado de motivación de la paciente; gran parte del éxito del tratamiento radica en el suficiente compromiso y participación de la paciente. ${ }^{46}$ En nuestra serie, la tasa de abandono fue de $14.2 \%$; es decir, 8 pacientes no completaron el programa por falta de motivación o ausencia de mejoría subjetiva. Debe evitarse ofrecer este tratamiento a pacientes desmotivadas y que, quizá, no vayan a cumplir y completar el programa.

La edad y paridad son los factores más estrechamente vinculados con la incontinencia urinaria de esfuerzo; se recomienda proponer el tratamiento conservador a pacientes que recientemente hayan parido, para evitar la aparición temprana de la incontinencia urinaria de esfuerzo. El TOT está contraindicado en mujeres con deseos reproductivos, por lo que la rehabilitación constituye el principal tratamiento en este grupo de pacientes.

\section{CONCLUSIONES}

La rehabilitación del piso pélvico, como tratamiento de la incontinencia urinaria de esfuerzo, se refleja en mejoría clínica de la incontinencia, como sucedió en nuestro estudio, a los seis meses de haber finalizado el tratamiento $83.3 \%$ de las pacientes tuvieron menos síntomas, disminución con la que se sintieron satisfechas con su calidad miccional; además, no requirieron corrección quirúrgica, lo que sucede en muy pocos casos.

\section{Financiamiento}

Los autores declaran no haber recibido algún patrocinio o financiamiento para llevar este estudio a cabo. 


\section{Conflicto de intereses}

Los autores declaran no tener conflicto de intereses.

\section{REFERENCIAS}

1. Moreno Sierra JGR, Prieto Nogal I, Fernández Montarroso S, Silmi Moyano, L. Etiologia, patogenia, tipos y clasificaciones de incontinencia urinaria y prolapsos urogenitales. In: Moreno Sierra J, editor. Atlas de incontinencia urinaria y piso pélvico. Madrid: ENE ediciones; 2007. p. 83-98.

2. Franco de Castro A, Espuña Pons M. Definiciones, clasificación y la incontinencia urinaria en números. Guías practicas de Urología Incontinencia urinaria. Madrid: Astellas; 2011. p. 1-8.

3. Offermans MP, Du Moulin MF, Hamers JP, Dassen T, Halfens RJ. Prevalence of urinary incontinence and associated risk factors in nursing home residents: a systematic review. Neurourol Urodyn. 2009;28(4):288-94.

4. Hannestad YS, Rortveit G, Sandvik H, Hunskaar S. A community-based epidemiological survey of female urinary incontinence: the Norwegian EPINCONT study. Epidemiology of Incontinence in the County of Nord-Trondelag. Clin Epidemiol. 2000;53(11):1150-7.

5. Ebbesen $\mathrm{MH}$, Hunskaar S, Rortveit G, Hannestad YS. Prevalence, incidence and remission of urinary incontinence in women: longitudinal data from the Norwegian HUNT study (EPINCONT). BMC urol. 2013;30(13).

6. Elia G, Dye TD, Scariati PD. Body mass index and urinary symptoms in women. Int Urogynecol J Pelvic Floor Dysfunct. 2001;12(6):366-9.

7. Hannestad YS, Rortveit G, Daltveit AK, Hunskaar S. Are smoking and other lifestyle factors associated with female urinary incontinence? The Norwegian EPINCONT Study. BJOG. Int J Gynaecol Obstet. 2003;110(3):247-54.

8. Hunskaar S, Arnold EP, Burgio K, Diokno AC, Herzog AR, Mallett VT. Epidemiology and natural history of urinary incontinence. Int Urogynecol J Pelvic Floor Dysfunct.. 2000;11(5):301-19.

9. MacLennan AH, Taylor AW, Wilson DH, Wilson D. The prevalence of pelvic floor disorders and their relationship to gender, age, parity and mode of delivery. Int J Gynaecol Obstet. 2000;107(12):1460-70.

10. Turner CE, Young JM, Solomon MJ, Ludlow J, Benness C. Incidence and etiology of pelvic floor dysfunction and mode of delivery: an overview. Dis Colon Rectum. 2009;52(6):1186-95.

11. Font Vilamitjana A. Exploración física. Valoración muscular del piso pélvico. In: Medica A, editor. Tratado de uroginecología Incontinencia urinaria. Barcelona: Espuña, M. Salinas, J.; 2004. p. 89-97.
12. Martínez Escoriaza JC. Clasificación de las disfunciones miccionales. Mecanismos de continencia. Fisiopatología de la incontinencia urinaria. In: Espuña Pons M, Salinas Casado J, editors. Tratado de uroginecología Incontinencia urinaria. Barcelona: Ars Medica; 2004. p. 31-9.

13. Petros PE, Ulmsten UI. An integral theory of female urinary incontinence. Experimental and clinical considerations. Acta Obstet Gynecol Scand. 1990;153:7-31.

14. Schafer W. Some biomechanical aspects of continence function. Scand J Urol Nephrol Suppl. 2001(207):44-60; discussion 106-25.

15. Alan C, Eren AE, Ersay AR, Kocoglu H, Basturk G, Demirci E. Efficacy of Duloxetine in the Early Management of Urinary Continence after Radical Prostatectomy. Curr Urol Rep. 2015;8(1):43-8.16.

16. McCormack PL, Keating GM. Duloxetine: in stress urinary incontinence. Drugs. 2004;64(22):2567-73; discussion 74-5.

17. Subak LL, Wing R, West DS, Franklin F, Vittinghoff $E$, Creasman JM, et al. Weight loss to treat urinary incontinence in overweight and obese women. N Engl J Med. 2009;360(5):481-90.

18. Newman DK. Conservative therapy for incontinence. In: Goldman HB, Vasavada SP, editors. Female Urology: a practical Clinical guide. Totowa, New York: Humana Press; 2007. p. 63-79

19. Wilson PD, GBerghmans B, Hagen S, Hay Smith J, Moore $\mathrm{K}$, al. E. Coservative management. In: Abrams $\mathrm{P}$, Cardozo $L$, Wein A, editors. Incontinence Basics \& evaluation 3rd International Consultation on Incontinence. 2. UK: Plymbridge distributions.; 2004. p. 855-964.

20. Bo K. Pelvic floor muscle training is effective in treatment of female stress urinary incontinence, but how does it work? Int Urogynecol J. 2004;15:76-84.

21. Bo K, Larsen SK, Osied S, al. e. Knowledge about and ability to correct pelvic floor muscle exercise in women with stress incontinence. Neurol Urodyn. 1988;7:261-2.

22. Bo K, Talseth T. Long-term effect of pelvic floor muscle exercise 5 years after cessation of organized training. Obstet Gynecol. 1996;87(2):261-5.

23. DeLancey JO. Structural aspects of the extrinsic continence mechanism. Obstet Gynecol. 1988;72(3 Pt 1):296-301.

24. Espuña Pons M, Pérez González A, Palau Pascual MJ, Puig Clota M. Tratamiento conservador. In: Franco de Castro A, Espuña Pons M, editors. Guías prácticas en urología Incontinencia urinaria femenina. Madrid.: Elsevier. Astellas.; 2011. p. 35-51.

25. Kegel A. Progressive resistance exercise in the functional restoration of the perineal muscles. An J Obst Gyn. 1948;56:238-48.

26. Berghmans LC, Frederiks CM, de Bie RA, Weil EH, Smeets LW, van Waalwijk van Doorn ES, et al. Efficacy of biofeedback, when included with pelvic floor muscle exercise 
treatment, for genuine stress incontinence. Neurourol Urodyn. 1996;15(1):37-52.

27. Berghmans LC, Hendriks HJ, Bo K, Hay-Smith EJ, de Bie RA, van Waalwijk van Doorn ES. Conservative treatment of stress urinary incontinence in women: a systematic review of randomized clinical trials. Br J Urol. 1998;82(2):181-91.

28. Glavind K, Laursen B, Jaquet A. Efficacy of biofeedback in the treatment of urinary stress incontinence. Int Urogynecol J Pelvic Floor Dysfunct. 1998;9(3):151-3.

29. Morkved S, Bo K, Fjortoft T. Effect of adding biofeedback to pelvic floor muscle training to treat urodynamic stress incontinence. Obstet Gynecol. 2002;100(4):730-9.

30. Hay-Smith EJ, Bo Berghmans LC, Hendriks HJ, de Bie RA, van Waalwijk van Doorn ES. Pelvic floor muscle training for urinary incontinence in women. The Cochrane database of systematic reviews. 2001(1):CD001407.

31. Kemmler W, Schliffka R, Mayhew JL, von Stengel S. Effects of whole-body electromyostimulation on resting metabolic rate, body composition, and maximum strength in postmenopausal women: the Training and ElectroStimulation Trial. J Strength Cond Res. 2010;24(7):1880-7.

32. Laycock J, Vodusek D. Electrical Simulation. In: Laycock J, Haslam J, editors. Therapeutic Management of Incontinence and Pelvic Pain. Londo: Springer; 2002. p. 85-9.

33. Gunnemann A, Heleis W, Pohl J, Paliakoudis I, Thiel R. The transobturator tape (TOT). A minimally-invasive procedure for the treatment of female urinary stress incontinence. Der Urologe Ausg A. 2004;43(9):1106-10.

34. Lim JL, Quinlan DJ. Safety of a new transobturator suburethral synthetic sling (TVT-O) procedure during the training phase. J Obstet Gynaecol Can. 2006;28(3):214-7.

35. Potic M, Ignjatovic I, Basic D. Tension free vaginal tape (TVT) vs Transobturator tape (TOT) complications and outcomes. Acta Chir lugosl. 2014;61(1):81-4.

36. Ulmsten $U$, Falconer $C$, Johnson $P$, Jomaa $M$, Lanner $L$, Nilsson CG, et al. A multicenter study of tension-free vaginal tape (TVT) for surgical treatment of stress urinary incontinence. 1998;9(4):210-3.
37. Zugor V, Labanaris AP, Rezaei-Jafari MR, Hammerer $\mathrm{P}$, Dembowski J, Witt J, et al. TVT vs. TOT: a comparison in terms of continence results, complications and quality of life after a median follow-up of 48 months. Int Urol Nephrol. 2010;42(4):915-20.

38. Soltero González A, Campoy Martínez P, Barrero Candau $R$, Medrano Sánchez $E$, Pérez Pérez $M$, Rodríguez Pérez A. Tratamiento rehabilitador de la incontinencia urinaria femenina. Arch Esp Urol. 2002;55(9):1035-46.

39. Cody J, Wyness L, Wallace S, Clazener C, Kilonozo M, Stearns $\mathrm{S}$, et al. Systematic review of the clinical effectiveness and cost-effectiveness of tension-free vaginal tape for treatment of urinary stress incontinence. Health Technol Assess. 2003;7(21).

40. Castillón Vela IT, Redondo González E, Linares Quevedo AI, Vallejo Herrador J, Ríos González E, Sáenz Medina J, et al. Cirugia de la incontinencia urinaria femenina en régimen de cirugia mayor ambulatoria: estudio de impacto presupuestario. Arch Esp Urol. 2007;60(3):267-72.

41. Enraf Electroterapia EM. http://www.enraf.pt/documentacion/electroterapia.pdf. 2014. Consultado 2 mayo 2016.

42. Pardo Schanz J, Ricci Arriola P, Solà Dalenz V, Tacla Fernández X. Trans-Obturator-Tape (TOT) for the surgical repair of stress urinary. Arch Esp Urol.Madrid 2006;59(3).

43. Mayorga Gómez E, J G-S, J.A. H, Preciado DA, Sedano JE, Trujillo L, et al. Complicaciones de la colocación de malla sintética vaginal para la incontinencia urinaria de esfuerzo. Rev Mex Urol 2015;75(3):132-6.

44. Smith F, Holman C, Moorin R, al. e. Lifetime risk of undergoing surgery for pelvic organ prolapsed. Obstet Gynecol. 2010;116:1096-100.

45. Fialkow MF, Newton KM, Lentz GM, al. e. Lifetime risk of surgical management for pelvic organ prolapse or urinary incontinence. Int Urogynecol J Pelvic Floor Dysfunct. 2008;19:437-40.

46. Pascual Amorós MA, Pedrosa Guerra Al. Tratamiento conservador de la incontinencia urinaria. In: Castro Dias D, Espuña Pons M, editors. Tratado de incontinencia urinaria. 1. Madrid: Luzan; 2006. p. 463-98. 